\title{
Experimental study on demulsification speed of emulsified asphalt based on equivalent surface area principle
}

\author{
Yan $\mathrm{Li}^{1}$, Hao Liang ${ }^{2}$, Jiangtao Lin ${ }^{2 *}$, Liang Fan², Yongzhen $\mathrm{Li}^{2}$, and Hui Wei ${ }^{2}$ \\ ${ }^{1}$ Shandong Hi-speed Company Limited, Jinan, China \\ ${ }^{2}$ Shandong Transportation Institute, Jinan, China
}

\begin{abstract}
In order to simplify the demulsification speed test of emulsified asphalt, rapid-breaking, medium-breaking and slow-breaking emulsified asphalt as well as different aggregates were selected for the demulsification speed test. The specific surface area of each single grade was determined by the actual measurement, and the equivalent aggregate was collected according to the principle of equivalent surface area. The results show that the equivalent aggregate can be composed by the assembling of gradations smaller than $0.075 \mathrm{~mm}$ and one or two bigger gradations. Therefore, the types of gradation in the equivalent aggregate will be reduced by one-half compared with that in the standard aggregate. The surface area of the equivalent aggregate is the same as that of the standard aggregate, and under a certain volume ratio, the demulsification rate determined by equivalent aggregate or standard aggregate is the same.There is also a critical volume ratio existing for the equivalent aggregate, If the critical volume ratio is exceeded, the demulsification rate determined by the equivalent aggregate will be greatly different from that determined by the standard aggregate. It is also found that the volume ratio turns to 1 without any changes when the proportion of gradation smaller than $0.075 \mathrm{~mm}$ reaches $25 \%-30 \%$.
\end{abstract}

\section{Introduction}

The demulsification speed test of emulsified asphalt is to determine the mixing conditions between emulsion and aggregate, on the base of the covering homogeneity of emulsion film on the surface of the aggregate, which is an essential test for distinguishing the breaking type of a particular emulsified asphalt ${ }^{[1]}$. Multiply researches ${ }^{[2-6]}$ have been done to analyse the factors affecting the selfstability of emulsified asphalt, including the particle microscopic state, composition, electric potential structure, emulsifier types, preparation process and its thermal effect. $\mathrm{Xu} \mathrm{Jie}{ }^{[7]}$ considers that the essence of demulsification is the evaporation of water and the aggregation of particles. Based on the optical fiber analysis of CA mortar, Li Yunliang ${ }^{[8]}$ considers that the demulsification is the combination process of asphalt particles. Kong Lingyun ${ }^{[9-10]}$ believes that the demulsification speed could be strongly influenced by the acidity or the alkalinity of aggregate. He also established a method to evaluate the demulsification speed of emulsified asphalt by the utilization of both centrifugal field and gravity field conversion model. Li $\mathrm{Bo}^{[11]}$ studies the demulsification speed of different emulsified asphalt through electrodynamics, and puts forward the corresponding determination method.

Generally speaking, many fruitful results have been achieved on the research of demulsification speed mechanism, however, researches mentioned above put too much emphasis on the stability of emulsified asphalt itself or the demulsification mechanism. In particle applications, the mixing condition between emulsified asphalt and aggregate would be the first class of interest. In standard specification, five different gradations, which are $0-0.075 \mathrm{~mm}, 0.3-0.075 \mathrm{~mm}, 0.6-0.3 \mathrm{~mm}, 2.36-0.6 \mathrm{~mm}$ and $4.75-2.36 \mathrm{~mm}$, are used for the preparation of two different aggregates (A \& B) according to a certain proportion. Clearly, the standard test requires too much weighing in the process, which is both inconvenient and tedious. Therefore, simplification of demulsification speed test and improvement of efficiency are the crucial technical problems to be solved in current work.

In fact, demulsification happens when emulsified asphalt particles make contact with the aggregate. The specific surface area of aggregate represents the surface area per unit mass of aggregate that contact with the emulsified asphalt, which is an important factor affecting the demulsification speed. In this paper, the equivalent aggregate with simpler gradation was prepared for carrying out the demulsification speed test based on the principle of equivalent surface area.

\section{Material Selection}

\subsection{Emulsified Asphalt}

\footnotetext{
* Jiangtao Lin: linjiangtaokeyan@126.com
} 
In this paper, cationic rapid-breaking, medium-breaking and slow-breaking emulsified asphalt were selected to run the following tests, which are demulsification speed, ionic charge, viscosity, evaporation residue and storage stability, according to the technical requirements of
"Standard Test Methods of Bitumen and Bituminous Mixtures for Highway Engineering" (JTG E20-2011).

Test data results are shown in the Table 1. It should be noted that the emulsified asphalt used in this test will not be limited to the three types below.

Table 1. Test results of emulsified asphalt.

\begin{tabular}{|c|c|c|c|c|c|}
\hline \multicolumn{2}{|c|}{ Test subjects } & Unit & Sample 1 & Sample 2 & Sample 3 \\
\hline \multicolumn{2}{|c|}{ Demulsification speed } & - & slow-breaking & medium-breaking & rapid-breaking \\
\hline \multicolumn{2}{|c|}{ Ionic charge } & - & cationic & cationic & cationic \\
\hline \multicolumn{2}{|c|}{ Residue on sieve (1.18mm) } & $\%$ & 0 & 0 & 0 \\
\hline \multicolumn{2}{|c|}{ Standard viscometer C25,3 } & $\mathrm{s}$ & 18 & 19 & 21 \\
\hline \multirow{5}{*}{$\begin{array}{l}\text { Evaporation } \\
\text { residue }\end{array}$} & Residue content & $\%$ & 61 & 60 & 61 \\
\hline & Penetration $\left(25^{\circ} \mathrm{C}\right)$ & $0.1 \mathrm{~mm}$ & 52 & 51 & 56 \\
\hline & Softening point & ${ }^{\circ} \mathrm{C}$ & 54 & 52.5 & 51.3 \\
\hline & Ductility $\left(5^{\circ} \mathrm{C}\right)$ & $\mathrm{cm}$ & 31 & 29 & 33 \\
\hline & Solubility & $\%$ & 99.2 & 99.4 & 99.7 \\
\hline \multirow{2}{*}{$\begin{array}{l}\text { Storage } \\
\text { stability }\end{array}$} & $1 d$ & $\%$ & 0 & 0 & 0 \\
\hline & $5 \mathrm{~d}$ & $\%$ & 1.0 & 0.8 & 0.7 \\
\hline
\end{tabular}

\subsection{Aggregate}

The basalt aggregate was selected for the sieving test in accordance with the relevant requirements of "Standard Test Methods of Bitumen and Bituminous Mixtures for
Highway Engineering" (JTG E20-2011), in which the standard sieves are $4.75 \mathrm{~mm}, 2.26 \mathrm{~mm}, 1.18 \mathrm{~mm}, 0.6 \mathrm{~mm}$, $0.15 \mathrm{~mm}$ and $0.075 \mathrm{~mm}$, respectively. Aggregates bigger than $4.75 \mathrm{~mm}$ were screened out, and the passing rate for each sieve was calculated. Test results are shown below.

Table 2. Sieving results.

\begin{tabular}{cccccccc}
\hline Sieve $(\mathrm{mm})$ & 4.75 & 2.36 & 1.18 & 0.6 & 0.3 & 0.15 & 0.075 \\
Passing rate $(\%)$ & 100 & 64.1 & 44.4 & 28.6 & 16.3 & 12.6 & 8.9 \\
\hline
\end{tabular}

Generally, the standard aggregate in demulsification speed test is composed of several gradations, including $0-0.075 \mathrm{~mm}, 0.3-0.075 \mathrm{~mm}, 0.6-0.3 \mathrm{~mm}, 2.36-0.6 \mathrm{~mm}$, and $4.75-2.36 \mathrm{~mm}$. Each gradation might include one or two single grades. For example, $0.3-0.075 \mathrm{~mm}$ gradation includes two single grades, which are $0.3-0.15 \mathrm{~mm}$ and $0.15-0.075 \mathrm{~mm}$. Therefore, the sieving results could reveal the detail information towards the specific composition of each gradation.

\section{Equivalent Aggregate Preparation}

\subsection{Method Selection}

\subsubsection{Empirical method}

Before the preparation of equivalent aggregate, the specific surface area of standard aggregate should be calculated first. The calculation of specific surface area is generally divided into empirical method and actual measurement method. More specifically, there are two commonly applied empirical methods in our country, which are empirical formula proposed by the American Asphalt Institute and empirical formula from Australia, respectively. In American empirical formula, the total surface area coefficient of aggregate is obtained by multiplying the passing rate of each sieve by the surface area coefficient ${ }^{[11]}$. As for the Australia method, the specific surface area is calculated by both the given empirical surface area coefficient and the formulas which are provided in the 1996 "Asphalt Pavement Design Manual". Both of the above methods are cited in the "Technical Specifications for code for Construction of Highway Asphalt Pavement" (JTG F40-2004) in the chapter of asphalt mixture design. The specific calculation formulas are as follows:

$$
\begin{gathered}
S A=\sum\left(P_{\mathrm{i}} \times F A_{\mathrm{i}}\right) \\
S A=\left[2+\sum\left(P_{\mathrm{i}} \times F A_{\mathrm{i}}\right)\right] \times 0.20482
\end{gathered}
$$

In formula (1) and (2): SA stands for the specific surface area of aggregate; $P_{i}$ is the sieving passing rate for each grade; $F A_{i}$ is the surface area coefficient of each grade. Table 3 shows the surface area coefficients given by two empirical methods.

Table 3. Empirical surface area coefficient.

\begin{tabular}{ccccccccc}
\hline \multirow{2}{*}{ Index } & \multicolumn{8}{c}{ sieve size, $\mathrm{mm}$} \\
\cline { 2 - 9 } & $>4.75$ & 4.75 & 2.36 & 1.18 & 0.6 & 0.3 & 0.15 & 0.075 \\
\hline America FAi & 0.41 & 0.41 & 0.82 & 1.64 & 2.87 & 6.14 & 12.29 & 32.77 \\
Australia FAi & - & 0.02 & 0.04 & 0.08 & 0.14 & 0.3 & 0.6 & 1.6 \\
\hline
\end{tabular}




\subsubsection{Actual Measurement Method}

The specific surface area of aggregate can be also obtained by the actual measurement. For particles less than $0.075 \mathrm{~mm}$, the nitrogen adsorption method is usually adopted, while image analysis method can be applied for the particles that are larger than $0.075 \mathrm{~mm}$. The actual measurement results are shown in Table 4.

Table 4. Measured specific surface area.

\begin{tabular}{cccccccc}
\hline sieve size $(\mathrm{mm})$ & $2.36-4.75$ & $1.18-2.36$ & $0.6-1.18$ & $0.3-0.6$ & $0.15-0.3$ & $0.075-0.15$ & $<0.075$ \\
\hline $\begin{array}{c}\text { specific surface area } \\
\left(\mathrm{m}^{2} \mathrm{~kg}^{1}\right)\end{array}$ & 0.45 & 0.93 & 1.66 & 3.51 & 5.36 & 10.83 & 111.49 \\
\hline
\end{tabular}

\subsubsection{Comparative Analysis}

The standard aggregate for demulsification test are divided into standard $\mathrm{A}$ and standard $\mathrm{B}$, each of which is made up of different gradations according to a certain proportion.

Table 5 is the specific composition for standard A and standard B.

Table 5. Specific standard aggregate composition.

\begin{tabular}{|c|c|c|c|c|}
\hline No. & single grade $(\mathrm{mm})$ & gradation $(\mathrm{mm})$ & Standard A (\%) & Standard B (\%) \\
\hline 1 & $<0.075$ & $<0.075$ & \multirow{3}{*}{3} & 10 \\
\hline 2 & $0.15-0.075$ & \multirow{2}{*}{$0.3-0.075$} & & \multirow{2}{*}{30} \\
\hline 3 & $0.3-0.15$ & & & \\
\hline 4 & $0.6-0.3$ & $0.6-0.3$ & 5 & 30 \\
\hline 5 & $1.18-0.6$ & \multirow{2}{*}{$2.36-0.6$} & \multirow{2}{*}{7} & \multirow{2}{*}{30} \\
\hline 6 & $2.36-1.18$ & & & \\
\hline 7 & $4.75-2.36$ & $4.75-2.36$ & 85 & - \\
\hline
\end{tabular}

The standard A and B are both composed of four different gradations, and each grade contains one or two single grades. For instance, the $0.3-0.075 \mathrm{~mm}$ grade contains two different single grades, which are 0.075 $0.15 \mathrm{~mm}$ and $0.15-0.3 \mathrm{~mm}$ at a ratio of fifty to fifty; the $2.36-0.6 \mathrm{~mm}$ grade also contains two single grades, which are $0.6-1.18 \mathrm{~mm}$ and $1.18-2.36 \mathrm{~mm}$ with the composition ratio of fifty-five to forty-five. Therefore, the specific surface area of standard A and B shall be considered by both the sieving passing rate and composition proportion, which could be calculated according to the following formula.

$$
S A=\sum C_{\mathrm{i}} \times\left[\left(\sum P_{\mathrm{ij}} \times F A_{\mathrm{ij}}\right) / \sum P_{\mathrm{ij}}\right]
$$

In formula (3), SA represents the specific surface area; $\mathrm{C}_{\mathrm{i}}$ stands for the proportion of each gradation in the aggregate; $\mathrm{P}_{\mathrm{ij}}$ is the proportion of each single grade in one certain gradation; and $\mathrm{FA}_{\mathrm{ij}}$ indicates the surface area coefficient of each single grade. Combined with the sieving data and the composition of standard aggregates, the standard aggregate sieving results can be calculated (see Fig 1). Based on the data in Fig 1, Table 3 and Table 4, the specific surface area of standard aggregate could be calculated by both empirical method and actual measurement method respectively. Results are shown in Fig 2.

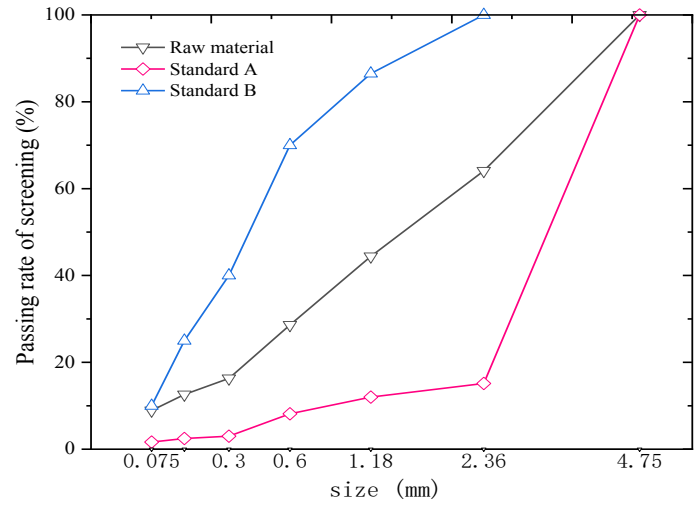

Fig. 1. Standard aggregate sieving results.

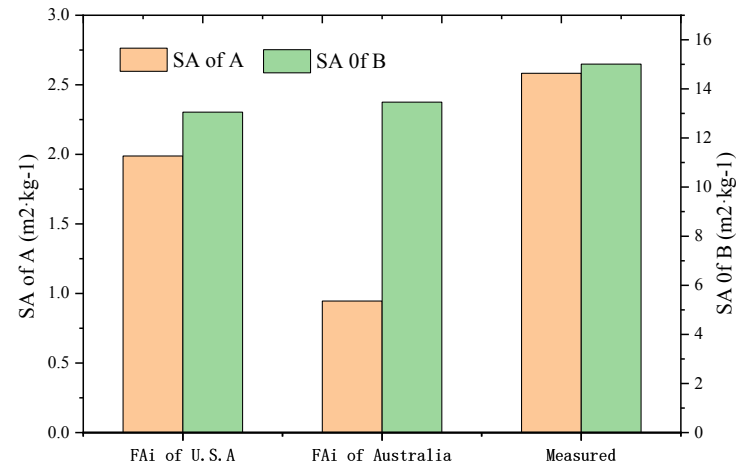

Fig. 2. Comparison of specific surface area.

Fig 1 shows that average particle size of standard A is much bigger than that of the standard $\mathrm{B}$, due to the different composition of gradations. From Fig 2, it can be seen that the specific surface area of standard A determined by the above three calculation methods are quite different. The results of standard B determined by 
the two empirical methods are basically the same, but both of them are smaller than the one determined by the actual measurement method. Analysis believed that the feasibility of the empirical formula lays on the fact that the minimum particle size of the gradations can be no more less than $0.03 \mathrm{~mm}^{[13]}$. However, in the real world, there is a large proportion existing for particles smaller than $0.03 \mathrm{~mm}$ in the gradations, especially for the 0 $0.075 \mathrm{~mm}$ gradation whose particle less than $0.03 \mathrm{~mm}$ can takes up to $60 \%$ or more. Since the specific surface area of gradation smaller than $0.03 \mathrm{~mm}$ accounts for a large proportion in the whole situation, the specific surface area of aggregate calculated by empirical method will be much smaller than the actual number. Therefore, in order to guarantee the accuracy for the following tests, this paper choose actual measurement method for the preparation of the equivalent aggregate. In the end, the specific surface areas of standard A and standard B are
$2.6 \mathrm{~m}^{2} \cdot \mathrm{kg}^{-1}$ and $15.0 \mathrm{~m}^{2} \cdot \mathrm{kg}^{-1}$ respectively, through the actual measurement calculation.

\subsection{Calculations and Preparations}

Compared with the standard aggregate, the equivalent aggregate should not only possess the same surface area, but also the identical detection validity.

In theory, the equivalent aggregate can be designed by various combinations of gradations according to certain mass and proportion, however, things are not necessarily the same. The preparation of equivalent aggregate shall be carried out in accordance with the determination standard of demulsification speed.

Table 6 exhibits the determination standard of demulsification speed.

Table 6. Determination standard of demulsification speed.

\begin{tabular}{cccc}
\hline Mixing condition for standard A & Mixing condition for standard B & Demulsification speed & Types \\
\hline $\begin{array}{c}\text { Non-union state of the mixture; } \\
\text { partics not fully covered with asphalt; } \\
\text { uneven asphalt distribution; } \\
\text { visible lumps in the mixture. }\end{array}$ & $\begin{array}{c}\text { Asphalt in the emulsion } \\
\text { immediately condenses into lump } \\
\text { once being mixed }\end{array}$ & Rapid-breaking & RS \\
Well-distributed mixture & $\begin{array}{c}\text { Non-union state of the mixture; } \\
\text { uneven asphalt distribution; visible } \\
\text { lumps in the mixture } \\
\text { Mushy state of the mixture; } \\
\text { even asphalt distribution }\end{array}$ & Medium-breaking & Slow-breaking \\
\hline
\end{tabular}

The demulsification speed is determined by the mixing state of both standard $\mathrm{A}$ and standard $\mathrm{B}$. Different gradations could lead to different mixing phenomena during the operation. Gradations larger than $0.075 \mathrm{~mm}$ could be used to estimate the non-union state of the mixture, meanwhile, the dispersion of the gradations smaller than $0.075 \mathrm{~mm}$ reflects the union state of the mixture. Therefore, the uniformity of mixing would be determined by both two parts mentioned above. And the equivalent aggregate shall be composed by both the gradation smaller than $0.075 \mathrm{~mm}$ and gradations larger than $0.075 \mathrm{~mm}$. The mass of the equivalent aggregate is as follows:

$$
m=(M \times S A) /\left(C_{\mathrm{i}} \times F A_{\mathrm{ij}}\right)
$$

In formula (4), m stands for the mass of equivalent aggregate, $M$ represents the mass of standard aggregate, SA is the specific surface area coefficient of standard aggregate, $\mathrm{C}_{\mathrm{i}}$ is the proportion of each gradation of aggregate, $\mathrm{FA}_{\mathrm{ij}}$ is the surface area coefficient of each gradation.

In this paper, the equivalent aggregate samples were prepared by a fixing proportion of equivalent $\mathrm{A}$ and $\mathrm{a}$ changing proportion of equivalent $B$.

Specific proportions are shown in the Table 7 , and Fig 3, 4, 5 exhibit some of the typical test results.

Table.7 Preparation of equivalent aggregate.

\begin{tabular}{ccc}
\hline Gradaion $(\mathrm{mm})$ & Equivalent A (\%) & Equivalent B (\%) \\
\hline$<0.075$ & 3 & $10-70$ \\
$0.6-0.3$ & 97 & $30-90$ \\
\hline
\end{tabular}

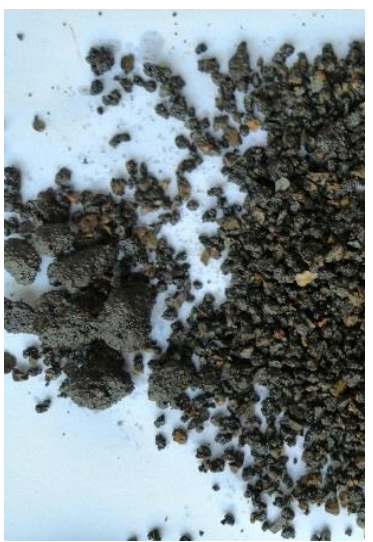

(a) Standard A

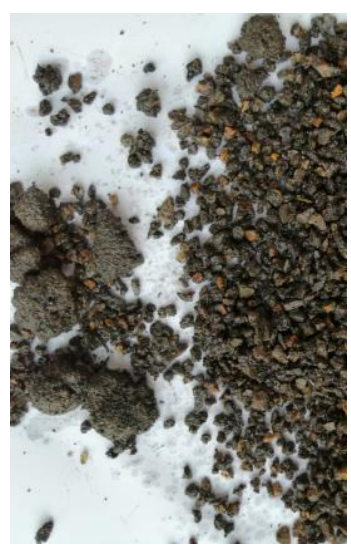

(b) Equivalent $\mathrm{A}$ 


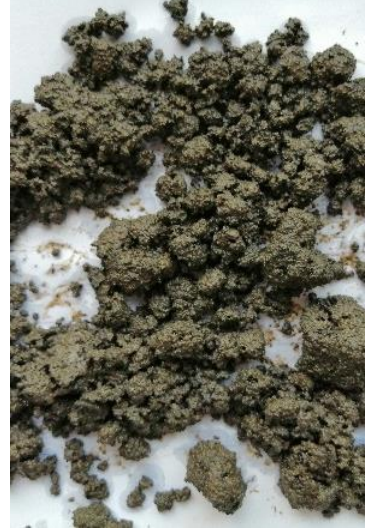

(c) Standard B

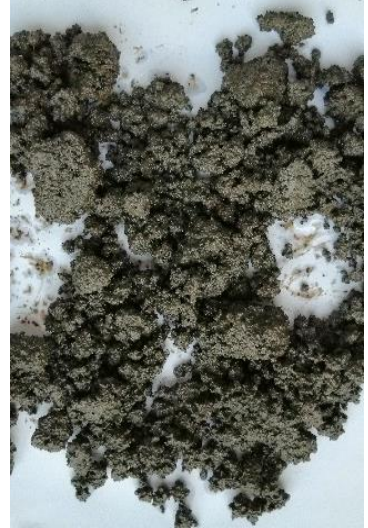

(d) Equivalent B
Fig. 3. Rapid-breaking emulsified asphalt.

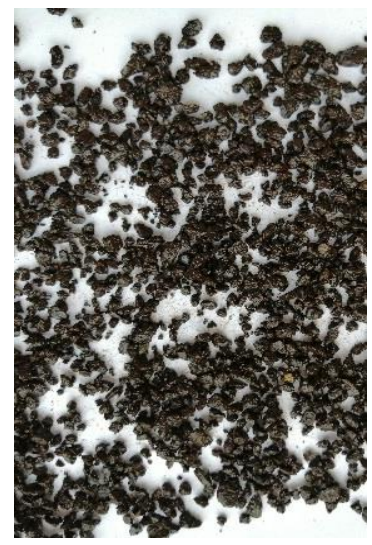

(a) Standard A

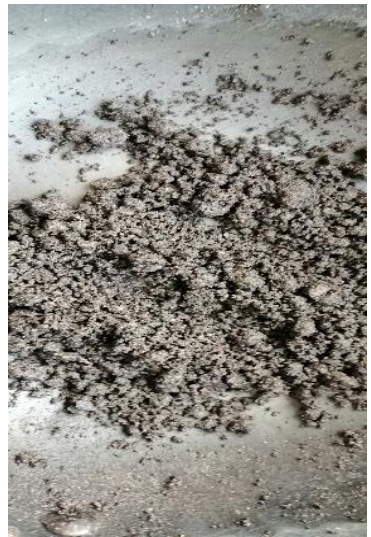

(c) Standard B

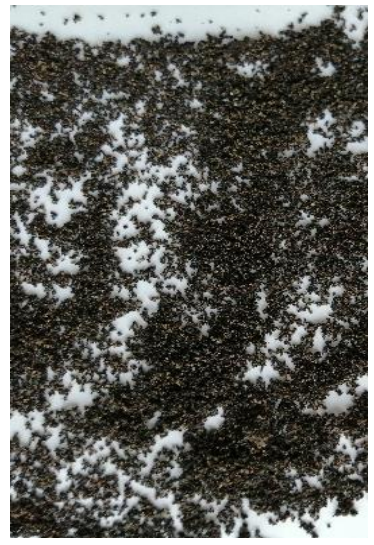

(b) Equivalent $\mathrm{A}$

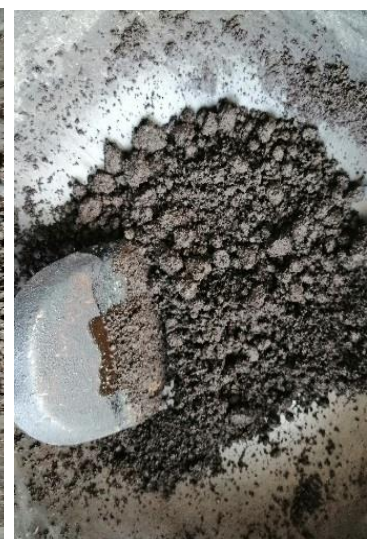

(d) Equivalent B
Fig. 4. Medium-breaking emulsified asphalt.

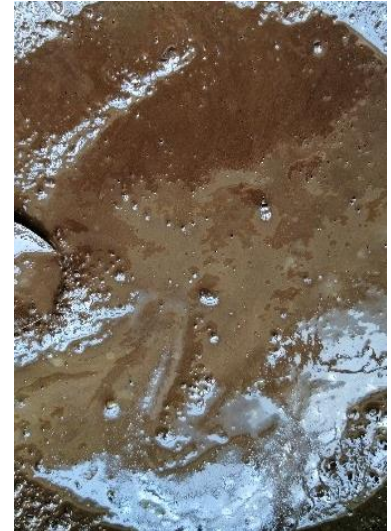

(a) Standard B

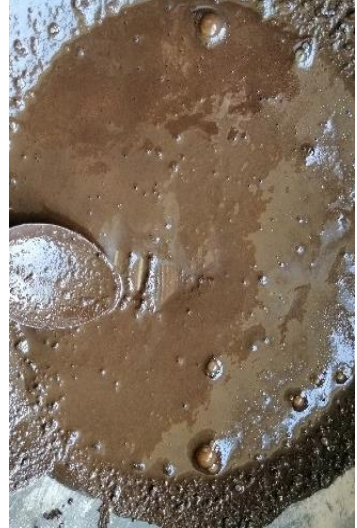

(b) Equivalent B
Fig. 5. Slow-breaking emulsified asphalt.

These validation test shows that the mixing condition as well as the determination of demulsification speed of equivalent aggregate with different proportion are the same as that of standard aggregate for most emulsified asphalt. However, it is found that the demulsification speed of medium-breaking emulsified asphalt changes when the proportion of gradation smaller than $0.075 \mathrm{~mm}$ exceeds $30 \%$. Specifically, during the mixing process of equivalent B versus medium-breaking emulsified asphalt, visible lumps start to appear at the very beginning and eventually turn into mushy state with further mixing, which could be misjudged as slow-breaking category according to this phenomenon. Analysis believes that with the increase proportion of gradation smaller than $0.075 \mathrm{~mm}$, the volume of the equivalent aggregate reduces gradually, which could be considered as the dilution of emulsified asphalt under the fixed amount of emulsion. This equivalent dilution will bring less effect to the demulsification speed within a certain range. However, over certain proportion, which we called critical concentration, the dilution effect will become extraordinarily noticeable, which will interfere with the clear judgment for the demulsification speed. This paper uses volume ratio to characterize the critical concentration, where volume ratio is the volume ratio of equivalent aggregate versus solid content of emulsified asphalt.

For further analysis, the composition detail of equivalent $\mathrm{B}$ and the volume ratio data were summarized towards samples who encountered the dilution phenomenon (in Fig 6). Fig 6(a) and 6(b) show that with the increase of the proportion of gradation smaller than $0.075 \mathrm{~mm}$, the mass changing rate of aggregate decreases; also, it can be seen from Fig 6(b) that the mass changing rate is close to zero when the proportion of gradation smaller than $0.075 \mathrm{~mm}$ reaches $30 \%$. In Fig 6(c), it is clear that the volume ratio holds the value of 1 when the proportion of gradation smaller than $0.075 \mathrm{~mm}$ stays at $30 \%$. Therefore, the critical volume ratio for this kind of emulsified asphalt is 1 , which means that the critical point to distinguish the demulsification state is when the equivalent aggregate volume is the same as the solid content volume of emulsified asphalt. 


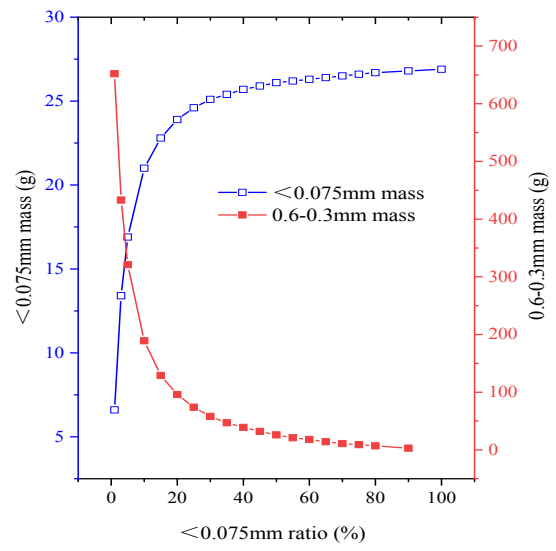

Fig. 6a. Diagram of mass changing.

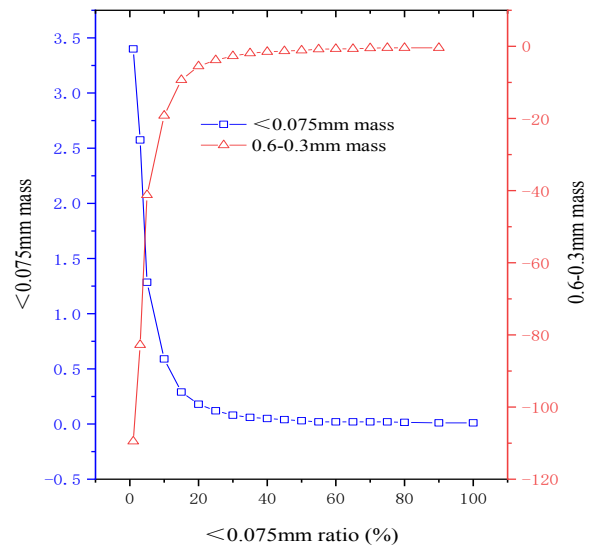

Fig. 6b. Diagram of mass changing derivative.

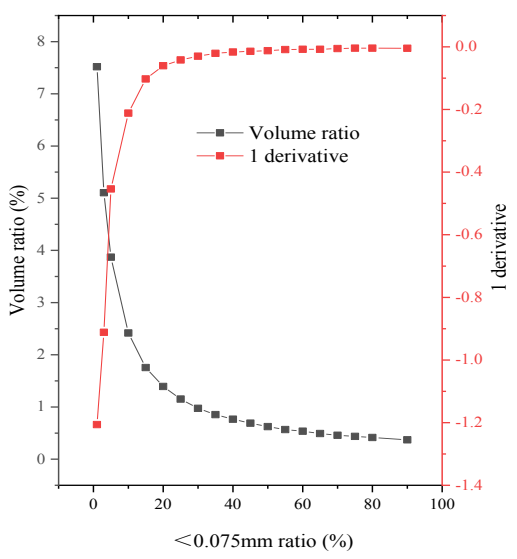

Fig. 6c. Diagram of volume ratio and its derivative.

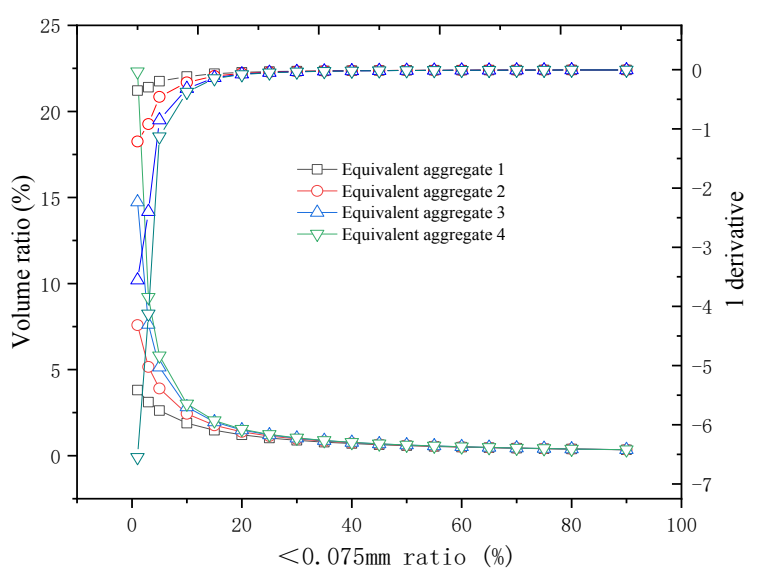

Fig. 7. Volume ratio and change rate of different equivalent aggregates.

The calculation of volume ratio and its changing rate of other equivalent aggregates are shown in Fig 7. The results show that different equivalent aggregates give different volume ratio even they have the same proportion of gradation smaller than $0.075 \mathrm{~mm}$. This is because that the volume ratio is determined by such factors as aggregate density, composition ratio, solid content and density of emulsified asphalt. However, with the increase of the proportion of gradation smaller than $0.075 \mathrm{~mm}$, the volume ratio diversity between different equivalent aggregates fades away gradually; until the proportion of gradation smaller than $0.075 \mathrm{~mm}$ reaches $25 \%-30 \%$, will the volume ratio be close to 1 without any changes. In fact, during practical application, the volume ratio basically stays at 1 when the proportion of gradation smaller than $0.075 \mathrm{~mm}$ is around $25 \%-30 \%$. Based on the above analysis, this paper draws the recommendation that the upper limit proportion of gradation smaller than $0.075 \mathrm{~mm}$ in the equivalent aggregate shall be set as $25 \%$, due to the emerge of misjudgement point of demulsification speed once the proportion of gradation smaller than $0.075 \mathrm{~mm}$ falls into $25 \%-30 \%$.

On the other hand, insufficient proportion of gradation smaller than $0.075 \mathrm{~mm}$ could also have adverse effects for the test. Fig 6(a) shows that insufficient proportion of gradation smaller than $0.075 \mathrm{~mm}$ will lead to an increase amount of material using for the test, bringing inconvenience in the process. It is also found that at the point when the proportion of gradation smaller than $0.075 \mathrm{~mm}$ is $10 \%$, the volume ratio of both standard aggregate and equivalent aggregate versus emulsified asphalt is basically 2.4. Therefore, the lower limit of proportion of gradation smaller than $0.075 \mathrm{~mm}$ in equivalent aggregate shall be set as $10 \%$ according to the specification.

Combined with the above analysis and the corresponding requirements of the specification, and for the purpose of ensuring the effectiveness and simplicity of the test.

The recommended selection is shown in Table8. 
Table 8. Selection of grades

\begin{tabular}{|c|c|c|c|c|}
\hline Gradation $(\mathrm{mm})$ & Standard A & Standard B & Equivalent $\mathrm{A}$ & Equivalent $\mathrm{B}$ \\
\hline$<0.075$ & \multirow{2}{*}{$\sqrt{ }$} & $\sqrt{ }$ & $\sqrt{ }$ & $\sqrt{ }$ \\
\hline $0.3-0.075$ & & $\sqrt{ }$ & o & o \\
\hline $0.6-0.3$ & $\sqrt{ }$ & $\sqrt{ }$ & $\sqrt{ }$ & $\sqrt{ }$ \\
\hline \multirow{2}{*}{$2.36-0.6$} & \multirow{2}{*}{$\sqrt{ }$} & \multirow{2}{*}{$\sqrt{ }$} & o & o \\
\hline & & & o & o \\
\hline $4.75-2.36$ & $\sqrt{ }$ & - & o & - \\
\hline
\end{tabular}

\section{Conclusion}

(1) The specific surface areas of both standard A and standard B determined by the empirical methods are smaller than the ones determined by the actual measurement method.

(2) The volume ratio of common aggregate versus emulsified asphalt is basically 1 without changing when the proportion of gradation smaller than $0.075 \mathrm{~mm}$ is around $25 \%-30 \%$. Some emulsified asphalt have their critical volume ratio, and the judgment of demulsification speed of emulsified asphalt will produce different results if the volume ratio exceeds the critical number.

(3) The recommended compositions of equivalent $\mathrm{A}$ and equivalent $\mathrm{B}$ were given in this paper. The types of gradation in the equivalent aggregate will be reduced by one-half compared with that in the standard aggregate, meanwhile, and under a certain volume ratio, the demulsification rate determined by equivalent aggregate or standard aggregate is the same.

It needs to be pointed out that the emulsified asphalt used in this test is a commonly used cationic emulsified asphalt. Further verification tests, as well as the relevant research about critical volume ratio, need to be carried out towards other types of emulsified asphalt.

\section{Acknowledgment}

The authors would like to thank the Shandong Technology Innovation Project (201731901168) for their support in this research.

\section{References}

1. JTG E20-2011, Standard Test Methods of Bitumen and Bituminous Mixtures for Highway Engineering.

2. $\quad$ L.S. Shi, X.X. Xin, Z.N. Guo, J. SD. Uni. 33(1), 97100(2003).

3. H. Feng, Y.G. Wang, Petro. Asphalt. 26(5), 3234(2012).

4. L.F. Zhu, AETD, 12, 4564(2018).

5. H.J. Shen, S.G. Hou, J. YZ. Uni. 22(2), 75-78(2019).

6. D.L. Yao, H.S. Zhou, New. Bui. Mat. 35(4), 8082(2008).

7. J. Xu, Ms. Thesis. NJ. Tech. (2017)
8. Y.L. Li, J. Ouyang, S.S. Wang, J. Har. Ins. Tech. 36(7), 997-1000(2015).

9. L.Y. Kong, F.L. Tang, Y. Xu, J. Highw. Transp. Res Dev. 33(10), 1-6(2016).

10. F.L. Tang, Ms. Thesis. CQ. JT. Uni. (2017)

11. B. Li, Y.B. Li, X. Liu, J. CN. F. Highw. 35(1), 279283(2015).

12. JTG F40-2004, Technical Specifications for code for Construction of Highway Asphalt Pavement.

13. D.B. Lv, H.B. Liu, C.L. Guan, J. Highw. Transp. Res Dev. 28(5), 28-32(2011). 